\title{
MÉTODO DE AMOSTRAGEM LARVAL DE Liriomyza sativae Blanchard (Diptera: Agromyzidae) EM MELOEIRO
}

\author{
Carlos Henrique Feitosa Nogueira ${ }^{1}$, Reginaldo Barros², Tiago Cardoso Costa Lima ${ }^{3}$, Valter Rodrigues \\ Oliveira ${ }^{4}$, Thiago Francisco de Souza Carneiro Neto ${ }^{5}$
}

\begin{abstract}
RESUMO - A mosca minadora Liriomyza sativae Blanchard é um inseto polífago, considerada umas das principais pragas do meloeiro na região semiárida brasileira. A definição de um método de amostragem é fundamental para a implementação de um programa de manejo integrado dessa praga. Sendo assim, o presente trabalho teve como objetivo definir a folha amostral para larvas de L. sativae na cultura do meloeiro. O estudo foi desenvolvido em duas áreas comerciais de melão no município de Juazeiro (BA). Após a emergência das plântulas, semanalmente realizou-se o monitoramento para acompanhar a ocorrência de mosca minadora até o término do ciclo. O ramo principal era observado para a presença de larvas de $L$. sativae, com a constatação, a folha era destacada e identificada a sua posição a partir da base do ramo. Sob microscópio óptico (40x) com luz por transmissão foi contabilizado o número de larvas vivas por folha. Foram realizadas sete coletas semanais a partir do décimo quinto dia após o plantio. Verificou-se que, de acordo com a fenologia da planta, difere a folha a ser amostrada. Para o monitoramento da mosca minadora deve-se amostrar as folhas da base para o ápice do ramo principal do meloeiro. Para as semanas 1, 2, 3, 4, 5 e 6 após o plantio, as folhas a serem amostradas devem ser a 1, 2 ou 3, 4, 6, 9, 14 e 20, respectivamente.
\end{abstract}

Palavras chave: controle, incidência, MIP, mosca minadora..

\section{SAMPLING METHODOLOGY FOR LARVAE OF LIRIOMYZA SATIVAE BLANCHARD (DIPTERA: AGROMYZIDAE) ON MELON PLANTS}

\begin{abstract}
The leafminer Liriomyza sativae Blanchard is a polyphagous insect, considered one of the main pests of melon in the Brazilian semiarid region. The definition of a sampling method is crucial for the implementation of an integrated management program for this pest. Therefore, this study aimed to define the sample sheet to L. sativae larvae in the melon crop. The study was conducted in two commercial areas of melon in Juazeiro (BA). After seedling emergence, weekly held monitoring to track the occurrence of leafminer fly until the end of the cycle. The main branch was observed for the presence of larvae of L. sativae, with the finding, the sheet was highlighted and identified its position from the branch base. Under optical microscope (40x) with light transmission was recorded the number of living larvae per leaf. Seven weekly collections were carried out from the fifteenth day after planting. It was found that, according to the plant phenology, differs from the sheet to be sampled. For monitoring the leafminer should be sampled leaves from the base to the apex of the main raw melon. For week 1, 2, 3, 4, 5 and 6 after planting, the sheets to be sampled should be 1 , 2 or 3, 4, 6, 9, 14 and 20, respectively.
\end{abstract}

Keywords: control, incidence, IPM, leafminer.

\footnotetext{
${ }^{1}$ Dr em Entomologia Agrícola, Professor do Departamento de Tecnologia e Ciências Sociais, Campus III, Universidade do Estado da Bahia, Av. Edgard Chastinet, s/n, Jardim Universitário, CEP: 48904-163, Juazeiro, BA, Brasil. E-mail: feitosa_nogueira@yahoo.com.br

${ }^{2}$ Dr em Entomologia, Professor do Departamento de Agronomia - Entomologia, Universidade Federal Rural de Pernambuco, Rua Dom Manoel de Medeiros, s/n, Dois Irmãos, 52171-900, Recife, PE, Brasil.

${ }^{3}$ Dr em Entomologia, Pesquisador da Embrapa Semiárido, Laboratório de Entomologia, Rodovia BR-428, Zona Rural, CEP: 56302-970, Petrolina, PE, Brasil.

${ }^{4}$ Dr em Genética e Melhoramento, Pesquisador da Embrapa Hortaliças, Laboratório de Melhoramento Genético, Embrapa Hortaliças, Rodovia BR-060, Km 09, CEP: 70351-970, Brasília, DF, Brasil.

${ }^{5}$ Graduando em Engenharia Agronômica, Departamento de Tecnologia e Ciências Sociais, Campus III, Universidade do Estado da Bahia, Av. Edgard Chastinet, s/n, Jardim Universitário, CEP: 48904-163, Juazeiro, BA, Brasil.
} 


\section{INTRODUÇÃO}

A mosca minadora Liriomyza sativae Blanchard (Diptera: Agromyzidae) é um inseto polífago, considerado praga primária em diversas culturas de importância econômica (Chandler \& Thomas 1983; Parrela 1987; Foster \& Sanchez 1988). Nos últimos anos tem sido o principal problema de ordem fitossanitário enfrentado pelos produtores de melão no Submédio do Vale do São Francisco (PE/BA), no polo MossoróAssu (RN) e no Baixo Jaguaribe (CE) (Araujo et al., 2007a; Costa-Lima et al., 2013).

Os danos ocasionados por moscas-minadoras no meloeiro são decorrentes da alimentação das larvas no mesófilo foliar, em que formam galerias reduzindo a área fotossintética da planta. Como consequência, os frutos apresentam baixo teor de sólidos solúveis totais ( ${ }^{\circ}$ Brix) (Araujo et al., 2007b). Após finalizar a alimentação larval, estas abandonam as folhas e passam pela fase de pupa no solo, onde irão emergir novos adultos (Minkenberg \& Lenteren, 1986).

Comumente os produtores de melão adotam apenas o método químico para o controle da mosca minadora, porém a decisão de quando realizar uma intervenção ainda é feita de maneira empírica, o que acarreta em falhas de controle bem como a elevação dos custos (Araujo et al., 2012). A adoção de um plano de amostragem para o controle de mosca minadora irá contribuir para que o produtor saiba o momento ideal de quando realizar a aplicação, conforme indicado por Trumble \& AlavaradoRodriguez (1992).

Através da determinação da unidade amostral que melhor represente a variação populacional de mosca minadora é possível ter uma estimativa precisa da densidade da praga para a tomada de decisão de controle (Gusmão, 2004).

Diante do exposto, o presente trabalho tem por objetivo determinar a unidade amostral para a amostragem de $L$. sativae na cultura do meloeiro tendo como base a contagem do número de larvas vivas durante todo o ciclo da cultura.

\section{MATERIALE MÉTODOS}

O presente trabalho foi realizado no período de Maio a Julho de 2015 em duas áreas comerciais de meloeiro, uma localizada na Fazenda Saraiva (Área I - Latitude $09^{\circ} 24^{\prime} 12,6^{\prime \prime}$ S, Longitude $40^{\circ} 24^{\prime} 42,1^{\prime \prime}$ e outra situada no Projeto Mandacaru I, município de Juazeiro (BA) (Área II - 9²3'19,3"S, 40²5'21,1").

\section{Áreas experimentais}

As áreas foram plantadas com sementes de melão da variedade Gladial, no dia 06-05-2015 (Área I) e no dia 12-05-2015 (Área II). Cinco dias após a emergência foi realizado o replantio na área, utilizando-se sementes da mesma variedade. A adubação da área foi de acordo com o manejo nutricional adotado pelo produtor. Não foi utilizada a manta agrotêxtil (TNT) para a proteção do cultivo.

O controle das pragas foi realizado com base apenas na utilização de inseticidas químicos, obedecendo a um calendário de aplicação utilizado pelo produtor. Nas duas áreas foram utilizados os inseticidas ciromazine, abamectina, imidacloprid e thiametoxan, utilizando as doses de bula registradas para a cultura e para o alvo, no Ministério da Agricultura, Pecuária e Abastecimento (MAPA).

\section{Condução do experimento}

Após a emergência das plântulas, foi realizado semanalmente o monitoramento das áreas para verificar o surgimento das primeiras larvas sobre as folhas verdadeiras. A lista com a idade das plantas e o número médio de folhas coletados por planta podem ser observados na Tabela 1.

Após o aparecimento das larvas na primeira folha verdadeira, semanalmente foi realizada a coleta de todas as folhas do ramo principal. Para a amostragem das plantas foi feito o caminhamento em zigue-zague, conforme

Tabela 1 - Dias após o plantio e número médio de folhas por planta de meloeiro no momento da coleta das folhas nas duas áreas. Maio a Junho de 2015, Juazeiro, Bahia.

\begin{tabular}{cc}
\hline Dias após o plantio & $\begin{array}{c}\text { No. médio de folhas } \\
\text { por ramo principal }\end{array}$ \\
\hline 7 & 1 \\
15 & 2 \\
22 & 4 \\
29 & 10 \\
34 & 12 \\
41 & 18 \\
47 & 23 \\
56 & 26 \\
\hline
\end{tabular}


recomendação de Guimarães et al. (2009), com distanciamento de $10 \mathrm{~m}$ por ponto. Semanalmente eram coletadas folhas de 40 plantas diferentes da semana anterior.

As folhas eram cortadas no pecíolo e acondicionadas em sacos de papel e transportadas para o laboratório. Sob microscópio estereoscópico (40x) com luz por transmissão, eram contabilizados o número de larvas vivas. O delineamento foi o de blocos ao acaso e os tratamentos foram representados pelas folhas que continham larvas vivas no dia da coleta, sendo que cada tratamento continha 40 repetições.

\section{Definição da folha a ser amostrada}

As contagens do número total de larvas vivas de $L$. sativae foram feitas aos 7, 15, 21, 28, 35, 42, 49 e 56 dias após o plantio (DAP). Os números médios de larvas vivas por folha foram comparados pelo teste de Scott-Knott a nível de $5 \%$ de probabilidade pelo Sorftware R (R Development Core Team, 2011).

\section{RESULTADOS E DISCUSSÃO}

Dos 7 aos 56 dias após o plantio, a rama principal do meloeiro variou de 01 a 26 folhas, respectivamente. Em ambas as áreas foram constatadas a presença de larvas de $L$. sativae a partir da segunda semana de avaliação. Foi observado que a mosca minadora tem preferência por ovipositar em folhas novas (Tabela 2).

A quantidade de folhas com larvas vivas foi ampliando na medida do crescimento da planta, variando de uma a sete folhas. De acordo com a fenologia da planta, variou a posição da folha com maior quantidade de larvas de mosca minadora. Considerando a folha a partir da base, foram estas: $1^{\text {a }}$ (15 DAP), $2^{\text {a }}$ ou $3^{\text {a }}$ (21 DAP), $4^{\text {a }}$ (28 DAP), 6 $6^{\text {a }}\left(25\right.$ DAP), 7 ${ }^{\text {a }} 8^{\text {a }}, 9^{\text {a }}$ ou $10^{\mathrm{a}}$ (42 DAP), $14^{\mathrm{a}}$ (49 DAP) e $19^{\mathrm{a}}$ ou $20^{\mathrm{a}}$ (56 DAP). De acordo com os resultados, não é possível padronizar uma folha para o monitoramento de $L$. sativae, visto que esta deve variar de acordo com a fenologia da planta.

Azevedo et al. (2005) também avaliaram a distribuição larval de $L$. sativae em folhas de meloeiro. No entanto, devido a diferenças metodológicas, torna-se difícil a comparação dos resultados. Os autores acompanharam área com cobertura agrotêxtil, avaliando apenas após 21 DAP, entre as folhas 10 e 20. Assim como, os mesmos não contabilizaram o número de larvas vivas, mas o número total de minas. Como forma de padronização,
Tabela 2 - Número médio de larvas vivas (média \pm erro padrão) de $L$. sativae em folhas de meloeiro. Área I de Maio a Junho de 2015, Área II de Maio a Julho, Juazeiro, Bahia.

\begin{tabular}{|c|c|c|}
\hline \multirow{2}{*}{ Folha $^{2}$} & \multicolumn{2}{|c|}{15 D. A. P1. } \\
\hline & Área I ${ }^{3}$ & Área $\mathrm{II}^{3}$ \\
\hline $1^{\mathrm{a}}$ & $3,2 \pm 0,27 \mathrm{a}$ & $5,1 \pm 0,37$ a \\
\hline $2^{a}$ & $\begin{array}{c}0,0 \pm 0,0 \text { b } \\
22 \text { D.A.P }\end{array}$ & $0,3 \pm 0,2 \mathrm{~b}$ \\
\hline $2^{a}$ & $2,0 \pm 0,48$ a & $4,2 \pm 0,35$ a \\
\hline $3^{\mathrm{a}}$ & $\begin{array}{c}2,7 \pm 0,50 \text { a } \\
29 \text { D.A.P. }\end{array}$ & $3,9 \pm 0,55 a$ \\
\hline $3^{a}$ & $1,8 \pm 0,22 \mathrm{c}$ & $2,4 \pm 0,39 \mathrm{c}$ \\
\hline $4^{\mathrm{a}}$ & $9,5 \pm 0,53$ a & $10,6 \pm 0,44 a$ \\
\hline $5^{a}$ & $\begin{array}{l}6,6 \pm 0,50 \text { b } \\
34 \text { D. A. P. }\end{array}$ & $5,4 \pm 0,15 b$ \\
\hline $4^{\mathrm{a}}$ & $4,1 \pm 0,28 \mathrm{c}$ & $3,3 \pm 0,38 c$ \\
\hline $5^{a}$ & $6,8 \pm 0,39 b$ & $7,9 \pm 0,29 b$ \\
\hline $6^{\mathrm{a}}$ & $8,7 \pm 1,04 \mathrm{a}$ & $10,7 \pm 0,44 a$ \\
\hline $7^{\mathrm{a}}$ & $\begin{array}{r}4,2 \pm 0,61 \text { c } \\
41 \text { D. A. P. }\end{array}$ & $4,9 \pm 0,51 \mathrm{c}$ \\
\hline $7^{\mathrm{a}}$ & $1,9 \pm 0,32 \mathrm{a}$ & $7,3 \pm 0,52 a$ \\
\hline $8^{a}$ & $2,7 \pm 0,50 \mathrm{a}$ & $8,6 \pm 0,30 a$ \\
\hline $9^{\text {a }}$ & $2,9 \pm 0,44 \mathrm{a}$ & $9,9 \pm 0,34 \mathrm{a}$ \\
\hline $10^{\mathrm{a}}$ & $\begin{array}{r}2,3 \pm 0,43 \text { a } \\
47 \text { D. A. P. }\end{array}$ & $7,1 \pm 0,63$ a \\
\hline $11^{\mathrm{a}}$ & $2,6 \pm 0,25 \mathrm{~d}$ & $4,5 \pm 0,23 \mathrm{~d}$ \\
\hline $12^{\mathrm{a}}$ & $7,6 \pm 0,55 \mathrm{c}$ & $10,3 \pm 0,45 c$ \\
\hline $13^{\mathrm{a}}$ & $10 \pm 0,55 b$ & $14,4 \pm 0,34 b$ \\
\hline $14^{\mathrm{a}}$ & $13,7 \pm 1,05 \mathrm{a}$ & $23,7 \pm 0,29 a$ \\
\hline $15^{\mathrm{a}}$ & $\begin{array}{c}8,7 \pm 0,50 \text { с } \\
56 \text { D. A. P. }\end{array}$ & $11,7 \pm 0,68 \mathrm{c}$ \\
\hline $16^{\mathrm{a}}$ & $3,27 \pm 0,37 d$ & $2,67 \pm 0,27 d$ \\
\hline $17^{\mathrm{a}}$ & $11,2 \pm 0,68 \mathrm{c}$ & $16,3 \pm 0,61 c$ \\
\hline $18^{\mathrm{a}}$ & $13,0 \pm 0,87 b$ & $14,5 \pm 0,38 b$ \\
\hline $19^{\mathrm{a}}$ & $17,0 \pm 0,84 \mathrm{a}$ & $21,4 \pm 0,97 a$ \\
\hline $20^{\mathrm{a}}$ & $15,4 \pm 0,83 a$ & $19,3 \pm 0,91 a$ \\
\hline $21^{\mathrm{a}}$ & $10,1 \pm 0,67 \mathrm{c}$ & $14,9 \pm 0,87 \mathrm{c}$ \\
\hline
\end{tabular}

${ }^{1}$ Dias Após o Plantio; ${ }^{2}$ Folha verdadeira; ${ }^{3}$ Médias seguidas pela mesma letra nas colunas não diferem pelo teste de Sott \& Knott a 5\% de probabilidade.

recomendaram a avaliação da $12^{\mathrm{a}}$ folha, entre 35 e 63 DAP, mesmo esta não sendo a folha mais infestada em todas as semanas. Levins et al. (1975) ressaltam que a contagem de minas pode se referir a danos anteriores, desse modo, reduzindo a precisão do método.

Dentre as semanas de avaliação, a sexta (41 DAP), foi a que apresentou maior número de folhas com médias similares de larvas de $L$. sativae, da sétima à décima. No entanto, as larvas presentes na folha 10 eram de ínstares iniciais e das folhas 7 e 8 muito desenvolvidas, 
próximas a sair da folha. Desta forma, considera-se que a folha 9 foi a mais representativa para sexta semana em virtude de ter larvas de primeiro e segundo instar. Para a terceira (22 DAP) e oitava semana (56 DAP) foi observado duas folhas com infestações similares. Para estes casos, como recomendação para padronização, sugerimos adotar o maior valor absoluto, no caso, a folha 3 (semana 3) e 20 (semana 8), visto que nessas folhas as larvas presentes eram de primeiro e segundo instar.

Pôde-se observar que as fêmeas de L sativae preferiram ovipositar em folhas mais novas. Este comportamento também foi observado por Azevedo et al. (2005) na cultura do meloeiro e por Dove (1985) na cultura da batata. Estes autores relatam que esta atração se dá pela facilidade do inseto em visualizar as folhas novas e ao crescimento vegetativo da planta. Minkenberg \& Ottenheim (1990) verificaram que $L$. trifolii apresentou preferência para se alimentar e ovipositar em folhas novas de tomateiro, em virtude destas apresentarem uma epiderme menos espessa em comparação com as folhas mais velhas.

De acordo com Braga Sobrinho et al. (2011), a folha a ser amostrada no meloeiro é a folha mais desenvolvida da rama, evitando as folhas em senescência. Porém, essa recomendação pode induzir ao produtor ao erro, visto que, foi observado que nas folhas mais desenvolvidas todas as larvas estavam desenvolvidas, próximas a saírem das folhas para pupação. Além do fato dos inseticidas registrados para o controle de moscas-minadoras possuírem eficiência maior em larvas pequenas de primeiro e segundo ínstares (Araujo et al., 2012).

Na área comercial em que se realizou o experimento, o produtor adotou o controle químico por calendário, aplicando semanalmente abamectina e ciromazina direcionados à L. sativae. Mesmo assim, verificouse que a média de larvas ultrapassou o nível de controle recomendado (Guimarães et al., 2008) de 5 larvas, em quatro das sete avaliações. Considerando o uso contínuo de inseticidas, sem adoção de monitoramento prévio, é provável a existência de populações resistentes aos produtos químicos aplicados. Há vários casos de resistência de Liriomyza spp. a vários princípios ativos (Sanderson et al., 1989; Parrella \& Trumble ,1989; Fergusson, 2004; Nadagouda et al., 2010).

Os resultados do presente trabalho irão colaborar para a realização de um monitoramento de $L$. sativae mais preciso na cultura do meloeiro, podendo auxiliar em programas de manejo integrado da praga na cultura, assim como, para definição de metodologia para estudos com a mosca minadora em condições de campo.

\section{CONCLUSÃO}

A unidade amostral a ser utilizada na amostragem da mosca minadora na cultura do meloeiro nas condições de plantio sem o uso do TNT aos 15, 21, 28, 35, 42, 49 e 56 dias após o plantio é a $1^{\mathrm{a}}, 2^{\mathrm{a}}$ ou $3^{\mathrm{a}}, 4^{\mathrm{a}}, 6^{\mathrm{a}}, 9^{\mathrm{a}}$, $14^{\mathrm{a}}$ e a $20^{\mathrm{a}}$ ou $21^{\mathrm{a}}$ folha verdadeira da rama principal, respectivamente, considerando as folhas da base para o ápice da planta.

\section{LITERATURACITADA}

ARAUJO, E.L.; COSTA, E.M.; MOURA FILHO, E.R. et al. Efeito de inseticidas sobre a mosca minadora (Diptera: Agromyzidae), quando aplicado durante a fase de ovo. Agropecuária Científica no Semi-árido, v.8, p.18-22, 2012.

ARAUJO, E.L.; FERNANDES, D.R.R.; GEREMIAS, L.D. et al. Mosca minadora associada à cultura do meloeiro no semi-árido do Rio Grande do Norte. Revista Caatinga, v.20, p.210-212, 2007a.

ARAUJO, E.L.; PINHEIRO, S.A.M.; GEREMIAS, L.D. et al. Técnica de criação da mosca minadora Liriomyza trifolii (Burgess) (Diptera:

Agromyzidae). Cpo. Digital, v.2, p.22-28, 2007b.

AZEVEDO, F.R.; GUIMARÃES, J.A.; TERAO, D. et al. Distribuição vertical de minas de Liriomyza sativae Blanchard, 1938 (Diptera: Agromyzidae) em folhas de meloeiro, em plantio comercial. Revista Ciência Agrícola, v.36, p.322-326, 2005.

CHANDLER, L.D.; THOMAS, C.E. Seasonal population trends and foliar damage of agromyzid leafminers on cantapoup in the Lower Rio Grande Valley, Texas. Journal of the Georgia Entomological Society, v.18, p.112-120, 1983.

COSTA-LIMA, T.C.; BUENO, V.H.P.; ALENCAR, J.A. Pragas de meloeiro. Revista Plasticultura, v.18, p.8-11, 2013.

DOVE, J.H. The Agromyzidae leaf miner, Liriomyza trifolii (Burgess), a new pest of potatoes and other vegetable crops in Mauritius. Acta Horticulturae, v.153, p.207-218, 1985. 
FERGUSON, J.S. Develpoment and stability of insecticide resistance in the leafminer Liriomyza trifolii (Diptera: Agromyzaidae) to cyromaine, abamectin, and spinosad. Journal of Economic Entomology, v.97, p.112-119, 2004.

FOSTER, R.E.; SANCHEZ, C.A. Effect of Liriomyza trifolii (Diptera, Agromyzidae) larval damage on growth, yield and cosmetic quality of celery in Florida. Journal of Economic Entomology, v.81, p.1721-1725, 1988.

GUIMARÃES, J.A.; BRAGA SOBRINHO, R.; AZEVEDO, F.R. et al. Manejo integrado de pragas do meloeiro, In: BRAGA SOBRINHO, R.; GUIMARÃES, J.A.; FREITAS, J.A.D.; TERÃO, D. (eds) Produção integrada de melão. Fortaleza: EMBRAPA Agroindustrial Tropical, 2008. p.183-199.

GUIMARÃES, J.A.; FILHO, M.M.; OLIVEIRA, V.R. et al. Biologia e manejo de mosca minadora no meloeiro. Fortaleza: Embrapa Agroindustrial Tropical, 2009. 9p. (Circular Técnica 77).

GUSMÃO, M.R. Amostragem de Liriomyza trifolii (Burgess) (Diptera: Agromyzidae) no tomateiro. Tese (Doutorado em Entomologia), UFV, Viçosa, 2004. 97p.

LEVINS, R.A; POE, S.L.; LITTELL, R.C.; JONES, J.P. Effectiveness of a leafminer control program for Florida tomato production. Journal of Economic Entomology, v.68, p.772-774, 1975.

MINKENBERG, O.P.J.M.; OTTENHEIM, J.J.G.W. Effect of leaf nitrogen content of tomato plants on preference and performance of a leafmining fly. Oecologia, v.83, p.291-298, 1990.
MINKERBEG, O.P.J.M.; LENTEREN, J.C.V. The leafminers Liriomyza bryoniae and $L$. trifolii (Diptera: Agromyzidae), their parasites and host plants: a review. Agricultural University Wageningen Papers, v.86, p.1-50, 1986.

NADAGOUDA, S.; PATIL, B.V.; VENKATESHALU; SREENIVAS, A.G. Studies on development of resistance in serpentine leaf miner, Liriomyza trifolii (Burgess) (Agromyzidae: Diptera) to insecticides. Karnataka Journal of Agricultural Science, v.23, p.56-58, 2010.

PARRELLA, M.P. Biology of Liriomyza. Annual Review of Entomology, v.32, p.201-224, 1987.

PARRELLA, M.P.; TRUMBLE, J.T. Decline of resistance in Liriomyza trifolii (Diptera: Agromyzidae) in the absence of insecticide selection pressure. Journal of Economic Entomology, v.82, p.365-368, 1989.

R DEVELOPMENT CORE TEAM. R: A language and environment for statistical computing. R Foundation for Statistical Computing, Vienna, Austria, 2011. ISBN 3-900051-07-0, URL http://www.R-project.org/.

SANDERSON, J.P; PARRELLA, M.P.; TRUMBLE, J.T. Monitoring insecticide resistance in Liriomyza trifolii (Diptera: Agromyzidae) with yellow sticky cards. Journal of Economic Entomology, v.82, p.1011-1018, 1989.

BRAGA SOBRINHO, R.; GUIMARÃES, J.A; ARAUJO, E.L. et al. Manejo integrado de pragas do meloeiro. Fortaleza: Embrapa Agroindustrial Tropical, 2011. 19p. (Circular Técnica 143).

TRUMBLE, J.T.; ALVARADO-RODRIGUEZ, B. Development and economic evaluation of IPM program for fresh market tomato production in Mexico. Agriculture, Ecosystems \& Environment, v.43, p.267-284, 1992.

Recebido para publicação em 2/10/2018 e aprovado em 11/3/2019. 